XVI.

\title{
Bericht iiber die III. Versammlung der Vereinigung mitteldeutscher Psychiater und Neurologen in Jena am 1. Mai 1898.
}

Nachdem am Vorabend eine gesellige Vereinigung stattgefunden hatte, begann die I. Sitzung am 1. Mai um 9 Uhr Vormittags in der psychiatrischen Klinik.

Anwesend incl. Gäste die Herren:

Ackermann-Hochweitzschen, Alzheimer-Frankfurt a. M., Balser-Köppelsdorf, Binswanger-Jena, Böhmig-Dresden, Brodmann-Jena, Bruns-Hannover, Buch olz-Marburg, v. BuchkaAltscherbitz, Dansauer-Jena, Fischer-Dresden, Frank (Russland), Friedland-Pensa (Russland), Friedländer-Jena, Ganser-Dresden, Gebhardt-Jena, Geist-Zschadrass, GreitherBayreuth. Gross-Altscherbitz, Grossmann-Nietleben, Gumprecht-Jena, Gïntz-Erfurt, II änel-Halle, Höhl-Leipzig, Höniger-Halle, Hösel-Zschadrass, Hüfler-Chemnitz, IlbergSonnenstein, Köster-Leipzig, Ko the -Friedrichroda, Kra use-Jena, Krehl-Jena, Laudenheimer-Leipzig, Link-Halle, LochnerThonberg bei Leipzig, Lond on-Halle, Watthes-Blankenburg a.H., Matthes-Jena, Mayser-Hildburghausen, Meyer-Roda, MöbiusLeipzig, Müller-Jena, Opitz-Chemnitz, Paetz-Altscherbitz, Peliza eus-Suderode, Pli ilipp-Jena, Pierson-Coswig, Pi p powErfurt, Quensel-Leipzig, Ranniger-Sonnenstein, Rebs-Gotha, Römer-Elsterberg, Sänger-Hamburg, Schaumburg-Altscherbitz, Schlegel-Untergötzsch, Schultes-Jena, Schwarz-Leipzig, Stintzing-Jena, Strubell-Jena, Tecklenburg-Coswig, T'euscher-Dresden, Verworn-Jena, Walsh-Philadelpbia, WardaBlankenburg i. Th., Weidhaas-Oberhof, Wiederhold-Wilhelmshöhe, Wind scheid-Leipzig, Yamamoto-O sak (Japan), Z ie henJena, insgesammt 69.

Die Sitzung wird eröffnet durch den I. Geschäftsführer der Vereinigung, Herrn Hofrath Prof. Binswanger-Jena, welcher die anwesenden Herren 
begrüsst und die Mittheilung macht, dass der II. Geschäftsführer, Herr Geh. Rath Prof. Hitzig am Erscheinen verhindert sei und auch andere Herren (Jolly, Moeli, Mendel u. a.) ihre Theilnahme abgesagt hätten. In Folge dessen mussten einige Vorträge von der Tagesordnung abgesetzt werden, wofür andere, nachträglich angemeldete eingeschoben wurden.

Hofrath Binswanger entledigt sich ferner des Auftrages des Herrn Staatsminister Freiherrn v. Gross, die Versammlung im Namen der Grossherzogl. Sächsischen Regierung willkommen zu heissen und den Verhandlungen einen gedeilichen Erfolg zu wünschen, und dankt Herrn Geh. Reg.Rath Freiherrn v. Boineburg vom Ministerium des Innern für seine Anwesenheit in der Versammlung.

Als Vorsitzender für die Vormittagssitzung wird Herr Ganser-Dresden durch Acclamation gewählt, zu Schriftführen berufen die Herren KrauseJena und Laudenheimer-Leipzig.

Es beginnen sodann die Vorträge.

I. Herr Oppenheim-Berlin: Ueber Brachialgie und Brachialneuralgie.

Vortragender führt an der Hand seines statistisch von ihm verarbeiteten Materials aus, dass die Brachialneuralgie ein sehr seltenes Leiden bildet, dass es sich in den so gedeuteten Fällen entweder um eine ansgesprochene Neuritis oder um symptomatisehe Schmerzen in Folge eines centralen (bezw. Wirbel-, Rü̈ckenmarksleidens) oder in der Mehrzahl der Fälle um eine Brachialgie, eine Psychalgia brachii, d. h. um einen durch einen neurasthenischen, hysterischen oder psychopathischen Allgemeinzustand bedingten Nervenschmerz handele.

Der Vortrag wird an andercm Orte ausführlich erscheinen.

Discussion: Herr Hösel-Zschadrass erwähnt einen Fall, der hierher gehört. Es handelt sich um eine etwa 50jährige Frau, die an secundärem Schwachsinn nach Melancholie leidet. Dieso Patientin bekommt im Jahre 2-3 mal heftige Schmerzen im linken Arm mit Anschwellung desselben in toto. Dass diese Art Brachialneuralgie psychogener Natur ist, scheint dem Redner daraus hervorzugehen, dass nach Anwendung der Therapie der zielbewussten Vernachlässigung das Leiden nach 8-14 Tagen schwindet.

Herr Bruns-Hannover stimmt dem Vortragenden darin bei, dass eigentliche Brachialneuralgien sehr selten sind. Häufiger sind schon Neuralgien in den Gebieten gewisser Rückenmarksnerven (referred pains). Eine besondere Gruppe bilden die nur nächtlich eintretenden Schmerzen, manche beruhen wohl auf Circulationsstörungen durch schlechte Lage. Die BeschäftigungsNeuralgien, besonders der sogenannte Schreibe- und Klavierspielerkrampf, entstehen meist auf neurasthenischer Grundlage, gehören dann also zu Oppenheim's Brachialgien.

Herr Moebius-Leipzig stimmt Oppenheim ganz bei, er hat nie eine wirkliche Arm-Neuralgie gesehen, wie sie in den Lehrbüchern beschrieben werden. Offenbar bestehen Beziehungen zwischen den ron Oppenheim beschriebenen Schmerzen und der Akinesia algera. 
Herr Stintzing-Jena stimmt im Allgemeinen den Ausführungen des Vortragenden zu, weist aber auf die Schwierigkeiten einer scharfen Abgrenzung der Brachialneuralgie gegen die Neuritis brachialis hin. Abgesehen von den leicht abzugrenzenden Plexus-Neuritiden, bei denen sich bald motorische Ausfallserscheinungen einstellen, giebt es doch auch solche mit ausschliesslich sensiblen Reizerscheinungen, die das Gepräge der neuralgischen Anfälle an an sich tragen.

Herr Ziehen-Jena betont, dass Brachialgie auch als erstes Symptom eines Hirn-Tumors in dem später gelähmten Arm vorkommt, ferner dass zuweilen eine Complication mit und Abhängigkeit von Angiospasmen beobachtet wird.

Herr Sänger-Hamburg erklärt sich im Grossen und Ganzen mit den Ausfübrungen des Vortragenden einverstanden, möchte aber darauf hinweisen, dass man sehr vorsichtig in der Stellung der Diagnose eines rein functionellen Leidens sein soll, da sich ein Sarkom des Humeruskopfes ganz unter den Erscheinungen der: Brachialgie entwickeln kann. Zum Beleg berichtet er über zwei derartige Fälle.

Herr Oppenheim (Schlusswort): Ich freue mich der Zustimmung, die meine Ausführungen gefunden haken. Einzelne in der Discussion gemachte Bemerkungen führen mich noch zu folgenden Darlegungon: Den von Herrn Hösel mitgetheilten Fall rechne jch gewiss zur Brachialgie. Das von Herrn Bruns angegebene nächtliche Auftreten dieser Schmerzattaquen ist auch mir sehr belcannt, und die Deutung dieser Neuralgien hat mich viel beschäftigt. Ausser den zur Erklärung yon Bruns angeführten Momenten möchte ich noch ein anderes zur Erklärung heranziehen: Es giebt Norvenstörungen, die sich durch ihr Auftreten im Schlaf kennzeichnen. Analog der Epilepsia nocturna giebt es auch eine Neuralgia nocturna, die eben durch den Schlaf ausgelöst wird. Herpes zoster ist bei Brachialnenralgie eine recht seltene Erscheinung und wohl meist neuritischen Ursprungs. Die Erörterung der Beziehungen dieser Brachialgie zu der Möbius'schen Akinesia algera würden uns zu weit führen, doch habe ich nicht den Eindruck, dass es sich bier um identische Dinge handelt. Ohne Weiteres gebe ich zu und habe dies schon in meinem Vortrage hervorgehoben, dass die Brachialgie von den Beschäftigungs-Neuralgien nicht immer zu trennen ist, und dass es weiter sehr schwierig sein kann, die Differentialdiagnose zwisehen der Neuritis und Neuralgie zu stellen, namentlich wenn man dem frischen Fall gegenübersteht. Die Brachialgie bei Hirntumor habe ich von meiner Statistik ausgesehlossen.

Die Beziehungen ' der Brachialgie zu vasomotorischen Störungen sind so aufgefasst, dass die letzteren ein Symptom der ersteren sind, doch halte ich es mit Herrn Ziehen nicht für ausgeschlossen, dass die Schmerzen auch die Folgeerscheinungen vasomotorischer Störungen bilden können.

II. Herr Mayser-Hildburghansen: Beitrag zur Lehre von der Manie.

Vortragender geht aus von der Auffassung Kraepelin's von der Manie als einer constitutionellen Psychose, die zu den periodischen Formen des Irre- 
seins gehöre. Er weist darauf hin, dass die Periodicität der maniakalischen Anfälle seit Langem bekannt sei und führt hierfür Belege aus der Literatur an. Er will nicht bestreiten, dass Kra epelin darin Recht habe, dass die einfache, einmal im Leben auftretende Manie eine grosse Seltenheit sei, trägt aber Bedenken hinsichtlich der Richtigkeit uud Wahrheit der von Kraepelin bohaupteten klaren Erkenntniss des Wesens der Manie. Eine exacte Prüfung der Kraepelin'schen Auffassung sei nur möglich an der Hand eines umfangreicheil Krankenmaterials. Die Cardinalfrage sei die: Giebt es thatsächlich eine wenn auch vielleicht nur selten vorkommende im Laufe des Lebens einmal und ausschliesslich auftretende selbstständige Störung von den anerkannten Symptomen der Manie oder nicht? Die Angabe von Kraepelin, dass von 1000 Kranken, deren Schicksal er verfolgt habe, nur einer einmal an manischer Erregung erkrankt gewesen sei und nachher nicht wieder, schiene ja Kraepelin's Ansicht zu rechtfertigen. Dieser Schein werde jedoch zerstört durch einen Zusatz Kraepelin's wie den, dass derartigen Fällen dieselbe Stellung einzuräumen sei, wie dem während des Lebens ein einziges Mal auftretenden epileptischen Anfall.

Versuche zur Entscheidung der Frage durch Zusammenstellung von Krankengeschichten und Nachfragen sind schon früher gemacht worden von ran Erp Talmann Kip und von Hinrichson. Talmann fand unter 41 Fällen 36 periodische und 4, die einen einmaligen Anfall durchgemacht hatten. Hinrichsen fand unter 125 Fällen von Manie 17 sicher geheilte, 9 davon sind während 11-21 Jahren nach der Entlassung gesund geblieben und befanden sich in einem Alter von 36--95 Jahren.

Der Vortragende hat nun sämmtliche Krankengeschichten der Hildburghäuser Anstalt, ca. 2400, einer Durchsicht unterworfen. Er hat in seine Statistik nur solche Fälle aufgenommen, bei welchen keine Form geistiger Störung: vor der Aufnahme bestanden hatte, bei welchen er nach der Schilderung des Krankheitsverlaufs eine der rein manischen oder hypomanischen Formen mit Sicherheit zu erkennen vermeinte, und die als gänzlich oder vielleicht auch mit gelindem Defekt geheilt entlassen waren. Solche nmuthmasslicb geheilte einfache Manieen" fand er 59 unter 2400 Krankheitsfällen, also ungefähr 21/2 pCt. der Gesammtsumme. Briefliche Recherchen ergaben, dass 4 davon später wieder erlirankt waren (rückfällig bezl. circulär), 1 hysterisch war, 9 psychisch gesund geblieben, aber gestorben waren (1 durch Suicidium, 8 an somatischen Krankheiten 1-10 Jahre nach der Entlassung). Von 9 Fällen waren keine Nachrichten zu erhalten. Der Rest von 32 Kranken (16 Männer, 16 Frauen) ist bisher und noch zur Zeit als geistig gesund geschildert. Die Zeit seit der Entlassung aus der Anstalt beträgt bei 15 (8 Männer, 7 Frauen) 1-10 Jahre, bei 17 (8 M., 9 F.) 11--31 Jahre. Von den 8 Männern mit über 10jähriger Genesungsdauer ist einer bisher 11, einer 16, zwei 17, einer 18, zwei 19, einer 20 Jahre lang gesund geblieben. Der älteste darunter ist jetzt 64 Jahre alt und seit 19 Jahren von einem bypomanischen Anfall genesen. Von den 9 Frauen ist eine 13, eine 16, drei 15, eine 18, zwei bisher 31 Jahre lang von Rü̈ckfällen verschont geblieben. Die älteste derselben mit 
21 jähriger Genesungsdauer ist gegentwärtig 60 Jahre alt, die beiden vor 31 Jahren von ihrer Mania typica genesenen stehen zur Zeit im 48. bezw. 49. Lebensjahre.

Auf Grund dieser Untersuchungen kann Vortr. nur zum Schlusse kommen, dass die einfache, solitär im Leben auftretende Manie allerdings eine grosse

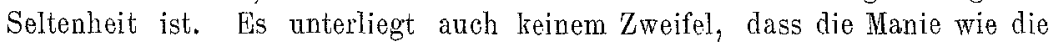
Melancholie in exquisiter Weise constitutionelle Psychosen sind, die gelegentlich bei einem und demselben Individuum innerhalb eines Zeitraumes von melreren Jahren mit einander abwechseln können, olhne damit ein circuläres Irresein einzuleiten. Unverständlich aber ist es dem Vortr. und nicht in Uebereinstimmung zu bringen mit dem sonst üblichen Gebrauch des Periodicitätsbegriffs, wenn Kräpelin die Manio ibrem Wesen nach als eine periodische Irrsinnsform bezeichnet. Er hebt aus seinem Material den Fall eines 12jährigen Knaben hervor, der 1878 eino 4 monatliche manische Erregung überstand, eine zweite 1879, bis heute aber gesund geblieben ist, ferner den Fall eines jungen Mädchens, das im 16. Lebensjahre Zeichen manischer Erregung bot, im darauffolgenden Jahre einen manischen Anfall ron der Dauer eines Jahres überstand und volle 18 Jahre nicht von Neuem erlirankte.

Mayser präcisirt schliesslich seine subjective Ueberzengung dahin, dass man die Manie zwar als eine constitutionelle Seelenstörung bezeichnen lö̈nne, welcher entsprechend der Natur ihrer Grundlage in ausserordentlich hohem Maasse die Nèigung zu Recidiven zukomme, dassman aber kein Recht habe, dieselbe ihrem Wesen nach schlechthin der Gruppe der periodischen Psychosen zuzuzählen.

Discussion: Herr Binswanger-Jena stimmt Herrn Mayser's Ausführungen zu und betont besonders das vereinzelte Auftreten ausgeprägter Manieen in der Entwickelungsperiode. Er theilt kurz den Fall einer Dame mit, welche im 19. Jahre eine typische Manie durchmachte und jetzt seit 13 Jahren trotz verschiedener Schädigungen (Puerperien u. a.) gesund geblieben ist.

III. Herr Sänger-Hamburg: Ueber Augenmuskelstörungen bei Hysterie. Vortr. hebt nach einem kurzen, historischen Ueberblick über den in Rede stehenden Gegenstand hervor, wie wenig geklärt noch die Ansichten über die bysterischen Bewegnngsstörungen des Auges siad. Am meisten geeignet zur Lösung der strittigen Fragen erscheine ihm die hysterische Ptosis, von der er die in der Literatur niedergelegten Fälle referirt und dann 8 eigene Beobachtungen an der Hand von Photographieen mittheilt.

Charcot und seine Schüler haben angenommen, dass die hysterische Ptosis stets durch einen Spasmus des Orbicularis, niemals durch eine Lähmung des Levator zu Stande komut. Die Fälle des Vortr, beweisen, dass Charcot's Ansicht nioht immer zutriff, und dass man in Uebereinstimmung mit Hitzig and Schmidt-Rimpler 2 Arten des Geschlossenseins des Lides bei der Hysterie zu unterscheiden babe, eine krampfhafte und eine schlaffe Form.

In charakteristischer Weise sieht man die spastische Form Charcot's in 2 seiner Fälle: Die eine Augenbrane steht tiefer als die andere, der freie 
Lidrand des betreffenden unteren Lides ist gehoben. Auf den Lidern sieht man den Lidrändern parallel verlaufende Fältchen. Die epitarsale, peritarsale und orbitale Partie des Orbicularis erscheint contrahirt, während der entsprechende Frontalis glatt ist.

In 4 weiteren Fällen bandelt es sich ebenfalls um spastische Formen der Ptosis, was an den querverlaufenden Fältchen und dem Höher- und Geradestehen des freien Randes des unteren Lides deutlich erkennbar ist. Jedoch stehen die Augenbrauen in beinahe gleicher Höhe und crscheinen in ihrer Krümmung nicht so different wie in den beiden ersten Fällen. Im Wesentlichen handelt es sich hier um einen Spasmus der epitarsalen Partie des Orbicularis. In einem Falle ist wohl auch der peritarsale theil mit inbegriffen.

In 2 Fällen endlich ist eine equisit schlaffe Ptosis zu constatiren. Schmidt-Kimpler's Ansicht, dass es sich in solchen Fällen um ein einfaches, willkürliches Erschlaffen des Levator palpebrae handelt, dürfte deshalb wohl nicht zutreffend sein, weil der Grad der Ptosis ein dauernd verschiedener auf keiden Seiten war. In der Deutung schliesst sich Vortragender der Hitzigschen Ansicht an, dass die hysterische Ptosis unter durchaus ähnlichen Umständen wie die übrjgen motorischen Innervationsstörungen der Kopfnerven, nämlich in der Regel auf Grund eines Reizungszastandes, aber in seltenen Fällen doch als Zeichen einer Lähnung zu Stande kommt.

Vortragender geht zum Schluss auf die neuerdings berichteten Pupillenstörungen bei der Hysterie ein. Der Annahme einer hysterischen Pupillenstarre auf Licht mit Mydriasis steht er absolut slieptisch gegenüber. Zweimal erlebte er solche Vorkommnisse, die sich bei genauor Untersuchung und Erkondigung als artificielle Mydriasis mit Sicherheit herausstellten, obwohl die betreffenden Hystericae hartnäckig geleugnet hatten. In einem 3. Fall ergab die Anamnese das Vorhandensein einer Lues. In don meisten Fällen dürfte es sich wohl um die Combination der Hysterie mit organischem Leiden des Centralnervensystems handeln. Sehr leicht ist ferner ein Irrthum möglich, indem ein langdauernder Spasmus iridis, wio er bei der Hysterie vorkommt, eine Lichtstarre vortäuschen kann. Bei genauer Untersuchung im Dunkelzimmer und Anwendung eines intensiven Lichts ist die Beweglichkeit der Iris mit Sicherheit zu constatiren.

Im hysterischen Anfall hat Vortragender noch nie eine reflectorische Lichtstarre der Pupille beobachtet, nur in einem Falle, wo er zweifelhaft war, ob es sich nicht doch un Epilepsie handele.

Discussion: Herr Schwarz-Leipzig weist hinsichtlich der Unterseheidung zwisehen reincr Ptosis und mit Contraction des Orbicularis verbundenem Lidschluss anf die ron Gowers hervorgehobene Erscheinung hin, dass bei Aufforderung, nach oben zu sehen, eine Contraction des Orbicularis sich durch leichte Lidzuckungen verräth. Reine, nicht mit Spasmus verbundene hysterische Ptosis fasst Schwarz nicht als eine dem willkürlichen Lidschluss analoge, nur eben hysterisch bedingte Erscheinung auf.

In der skeptischen Beurtheilung der "hysterischen Pupillenstörungen" schliesst sich Schwarz dem Vortragenden an, weist ferner auf die Wichtig. 
keit der Trennung zwischen reflectorischer und allgemeiner Pupillenstarre, sowie auf die Differentialdiagnose zwischen toxischer und anderweiter Pupillenlähmung mittelst Einträufelung von Pilocarpin oder Eserin hin und betont endlich den Werth genauer Accommodationsprüfung bei Fällen von anscheinend bysterischen Pupillenstörnngen.

Herr Oppenheim-Berlin erwähnt einen Fall von schlaffer Ptosis bei hysterischer Amaurose, den er schon früher beschrieben hat. Die reflectorische Pupillenstarre findet sich wohl auch bei Hysterie, ist aber kein Symptom derselben, sondern durch Lues, Tabes, Paralyse bedingt. Oppenheim erwähnt einen Fall, in welchem die reflectorische Pupillenstarre bei einer Hysterica 18 Jahre lang bestand, aber durch eine abgelaufene Hirn-Lues bedingt war.

Herr Bruns-Hannover nimmt an, dass die schlaffe "Ptosis" der Hysterischen auf unbewasst willliürlicher Aufhebung der Innervation des Augenlides, einem "Fallenlassen" des Lides beruht. Pupillenlähmung mit Mydriasis bei Hysterischen ist meist künstlich hervorgerufen, reflectorische Pupillenstarro ist, wenn sie bei Hysterischen rorkommt, eine organischo Complication.

Herr Stintzing-Jena stimmt nach seiner Erfahrung dem Vortragenden zu, dass es eine hysterische Ptosis im eigentlichen Sinne des Wortes giebt. Wie in jedem anderen motorischen Gebiete, so können bei Hysterie auch im Gebiete des Oculomotorius sowohl Krampf- als auch Lähmungserscheinungen auftreten. Ausgesprochene Pupillenstarre hat Stintzing bei Hysterie nicht gesehen; er ist der Ansicht, dass gerade das isolirte Auftreten der Ptosis unter Ausschluss der übrigen Oculomotoriuszweige bei Hysterie häufiger als bei organischen Erkrankungen und daher charakteristisch für Hysterie sei.

Herr Möbius-Leipzig bält streng an dem Satze fest: Es giebt keine hysterischen Augenmuskellähmungen; es giebt auch keine hysterische Ptosis. Möbius kann sich ganz Bruns anschliessell. Er macht auch darauf aufmerksam, dass die Einzelnen sehr verschiedene Geschicklichkeit im willkürlichen Schliessen der Lider laben. Ferner bittet Möbius dringend, den Ausdrurk reflectorische Pupillenstarre nur in dem Sinne Argyll-Rohertson's Symptom zu brauchen, d. h. Fehlen der Lichtreaction bei guter Convergenzverengerung. Dieses Symptom ist ausschliesslich der Tabes eigen.

Herr Sänger (Schlusswort) schliesst sich dem Wunsche des Herrn Schwarz an, dass bei den Pupillenuntersuchungen mehr auf die Accommodation geachtet werde. Er freut sich der Zustimmung von Seiten des Herrn Stintzing und wendet sich gegen die namentlich von Möbius vertretene Ansicht, dass alle Frscheinungen der Hysterie auf Vorstellungen beruhen. Vortragender behauptet, dass dies für manche Stigmata (concentr. Gesichtsfeldeinengung, Iriskrampf, circumscripte Analgesie auf dem Rücken etc.) gar nicht zutrifft. Vortragender weist darauf hin, dass, wenn wan Hysterische auffordert, die Oberlider zu erschlaffen, die Augenlider nicht glatt und gelähmt erscheinen, sondern meist das Rosenbach'sche Phänomen in Form von Vibriren der Lider zeigen. Was Bruns' Angabe vom Herabgehen der Oberlider beim Nachabwärtssehen betrifft, so ist dies keine Eirschlaffung, sondern beruht auf mechanisch-anatomischen Verhältnissen der Verbindung der Sehnen 
des Levator und Rectus superior, wie es deutlich beim Lagophthalmus zu Tage tritt.

Das von Oppenheim betonte Symptom des Zurückfallens der Obeslider beim Zurückbiegen des Kopfes hält Vortragender für eine Bereicherung der Untersuchungsmethoden behufs Stellung der Differentialdiagnose, ob Lähmung oder Spasmus besteht.

IV. Herr Warda-Blankenburg i. Thür.: Ueber degeneratjve Ohrformen.

Die Untersuchungen nach der 1895 von $\mathrm{Sch}$ walb e veröffentlichten Zällkarte umfassen 96 Männer und 87 Frauen der psychiatrischen Klinik zu Jena, functionelle Psychosen und angeborenen Schwachsinn, Epileptiker, Paralytiker und schwere Neurosen.

Form I der Darwin'schen Spitze (nach Schwalbe) fand sich überhaupt nicht, Form II plus Form III bei den Männern in $16,7 \mathrm{pCt}$., bei den Frauen in 22,4 pCt. der Ohren. Der Durchschnittsformwerth der Darwin'schen Spitze beträgt bei den Männern überhaupt 4, 3, bei den Nichtbelasteten 4,2 , bei den Belasteten 4,6; bei den Fraven lauten die entsprechenden Zahlen 4, 3, resp. 4, 6, resp. 4, 1. Der morphologische Ohrindex weist im Allgemeinen höhere Zahlen auf als bei Schwalbe. Die belasteten Männer und Frauen incliniren gegenuiber den nichtbelasteten zu etwas kleineren Werthen für diesen Index und zu etwas grösseren Werthen für den physiognomischen Ohrindex. Auf die Wange verlängerte Lobuli fanden sich bei den Männern in 7,8pCt. der Ohren, bei den Fraven in 11,1 pCt., einfach angewachseno Lobuli bei den Männern in $31,7 \mathrm{pCt}$., bei den Frauen in $40 \mathrm{pCt}$. aller Ohren. Erbliche Belastung scheint bei den Männern in dieser Beziehung bedeutungslos zu sein, bei den Fraven zeigen die erblich belasteten sogar ein besser entwickeltes Läppchen als die von Belastung freien. Zur Feststellung der degenerativen Bedeutung gewisser Ohrformen kommen ausser der Vergleichung von Geisteskranken mit Gesunden und ausser der Gegenüberstellung erblich Belasteter und Nichtbelasteter noch vergleichende Messungen in den Familien psychopathischer Individuen in Betracht.

V. Herr Teuscher-Dresden: Ueber suggestive Behandlung von Kindern.

Von einer Anzahl Autoren wird die hypnotische Behandlung der Kinder als schädlich oder doch wenigstens überflïssig hingestellt. Vortragender hält nach seiner Erfahrung die Hypnose im Kindesalter, wenn sie nur richtig ausgeübt wird, für vollständig gefahrlos und führt eine Anzahl Fälle an, in denen alle früher angewandten Methoden nicht zum Ziele gefïhrt hatten, die seitdem dauernd gesund geblieben sind. Das Alter der behandelten Kinder betrug 5 bis 11 Jahre. Unter ihnen wurden von 6 Bettnässern 5 geheilt und 2 Masturbanten von ihrem Laster befreit. Dann folgt die genaue Beschreibung der Behandlung eines Knaben, welcher den Typus eines jugendlichen Verbrechers zeigte, d. h. stahl, log, mit dem gestolilenen Gelde den grossen Herm spielte etc. Nach einem Recidiv ist der Knabe seit 2 Jahren gesund geblieben. Ein an hysterischen Dämmerungszuständen und Enuresis diurna et nocturna leidender 
Knabe hat seine Krankheitssymptome seit $1 \frac{1}{2}$ Jahren vollkommen verloren und entwickelt sich körperlich und geistig ausgezeichnet. Schliesslich erwähntVortragender noch ein Kind ron 5 Jahren, das an einer auf schlechter Angewöhnung beruhenden Sprachstörung litt, die in wenigen Sitzungen beseitigt wurde.

Da die angeführten Fälle zum Theil schon seit Jahren aus der Behandlung entlassen sind und sich eines dauernden Wohlbefindens erfreuen, glaubt Vortragender die volle Gefahrlosigkeit der suggestiven Belandlung von Kindern dargethan zu haben, die noch Erfolge aufweist, wo andere therapeutische Methoden nicht zum Ziele führten. Er ist mit Forel der Ansicht, dass die zielbewusste Anwendang dieser Methode von Pädagogen und Aerzten ( moralische Orthopädie") in Zukunft eine Bedeutung erlangen wird, deren Wichtigkeit noch gar nicht abgesehen werden kann.

Discussion: Herr Sänger-Hamburg ist gegen die Anwendung der Hypuose bei Kindern, da dieselben durch Wachsuggestion ebenso rasch und gut geheilt werden können und letztere ein harmloseres Mittel darstellt als die Hypnose. Bei hartnäckigen Fällen empfiehlt Sänger die Verbringung des Kindes in eine Anstalt.

VI. Herr Möbius-Leipzig: Psychiatrische Göthestudien.

Vortragender hat nicht die Person Göthe's, sondern vielmehr seine Werke zum Gegenstand seiner psychiatrischen Studien gemacht. Err weist darauf hin, dass sich in den Göthe'schen Werken viele pathologische Figuren finden, ebenso wie bei Shakespeare. Der Grund ist oflenbar der, dass beide Dichter einen "gegenständlichen Sinn" haben. Woher aber nahm nun Göthe seine Kenntnisse krankhafter Geisteszustände? In Anstalten, "Tollhäuser" wje er sic nannte, ging er nie, da schon genug" "Schwachköpfe und Narren" in der Welt herum. liefen. Er bildete sich vielmehr seine Ansichten durch Verlehr mit Personen und durch allgemeine Litteratur. Vortragender geht kurz auf die damaligen Bestrebungen der Irrenpflege (Gründung der Landesirrenanstalt in Jena 1804) ein, an denen Göthe keinon Antheil hatte. Dagegen finden wir zahlreiche pathologische Figuren in Göthe's Leben (Clauer, Lenz, Jerusalem, Frl. von Lassberg, Merk u. A.) und sehr viele, deren Charaktere an das Pathologische grenzten (seine Schwester Cornelia, Jung-Stilling, Herder, Lavater, Basedow ete.), Menschen, die Göthe sehr treffend beurtheilte. Krankhafte Geisteszuständo aus der Litteratur kennen zn lernen gab ihm Gelegenheit die Lectüre von Roussean, Tasso, Dante, der Bibel, des Benvenuto Cellini.

Vortragender geht dann auf die pathologischen Figuren in Göthe's Werlen ein. Er nennt als solche Werther, Gretchen, Orest, Mignon, den Harfner, Aurelia, Ottilie, Lenz, Zimmermann, Benvenuto Cellini, endlich den Tasso. Mit diesem beschäftigt sich Vortragender dann in seinen weiteren Ausführungen. Tasso ist ein geisteskranker Held, dem das normale, tragische Motiv fehlt. Er zeigt Symptome der Paranoia, gilt aber doch nicht als geisteskrank. Vortragender denkt sich die Entstehung der Figur des Tasso so, dass Göthe erst einen leidenschaftlichen Menschen zeichnen wollte, durch seine italienische Reise aber den historischen Tasso, der an Verlolgungswahnsinn und Hallucinationen litt, kennen lernte und dass anf diese Weise das Aesthetische in 
der Darstellung des Charakterbildes des Tasso gelitten habe. Möbius schildert näher den historischen Tasso und geht sodann auf den Göthe'schen näher cin, der sich im Allgemeinen als ein wunderlicher Mensch zeigt, der mit der Welt zerfallen ist.. In der Erregung aber fällt der Schleier und der beginnende Wahn tritt hervor. Schon das eine Citat, „es ist Verschwörung und Du bist das Haupt" kennzeichnet die Paranoia, deren Anfangsstadium Göthe mit grosser Feinheit geschildert hat. Göthe hat damit den Fehler begangen, Tasso als einen unheilbaren Geisteskranken zu charakterisiren, dessen Handlungen ausserhalb seiner Erregungen aber im Gegensatz zu seinen Wahnideen stehen. Vortragender glaubt, dass Göthe einen Grenzzustand im Auge gehabt liabe, sich aber über die Bedeutung des von ihm verwendeten historischen, krankhaften Zuges gotäuscht hat. Was Göthe mit der Schlusscene gewollt hat, weiss Niemand. Es kann sich jeder dabei denken, was ihm beliebt.

Discussion: Herr Oppenheim spricht sich principiell gegen die Verschwisterung psyohiatrischer und ästhetischer Betrachtungen aus, da die Psychiatrie nichts dabei gewinne und der ästhetische Genuss des Kunstwerkes höchstens eine Einbusse erleide. Tasso sei der mit höchstem Idealismus erfüllte Mensch, der nicht in die Welt der Wirklichleit hineinpasse und sich üborall an den Sachen stosse. Würde die Kunst sich darauf beschränken uns die Typen vorzuführen, die vor dem strengen Forum der Psychiatrie als normal gelten, so würde Vieles und vielleicht das Beste verloren gehen.

Es folgt die Wahl des Ortes für die nächste Versammlung. Einstimmig fällt die Wahl auf Dresden, ebenso werden einstimmig zu Geschäftsführern die Herren Ganser-Dresden and Pierson-Coswig gewählt.

Herr Ganser erstattet den Rechenschaftsbericht. Die Rechnungen werden von den Herren Paetz und Windschoid geprüft und richtig befunden. dls Kassenführer wird Herr Ilberg bestimmt. Zum Vorsitzenden für die Nachmittagssitzung wird Herr Mayser-Hildburghausen por Acclamation gewählt.

Nach der Frühstückspause beginnt um 1 Uhr die

\section{Sitzung.}

VII. Herr Laudenheimer-Leipzig: Ueber nervöse und psychische Störungen der Gummiarbeiter.

Die beim Vulkanisiren des Gummi (dureh Einathmung ron Schwefelkohlenstoff) entstehenden Vergiftungen kommen weit häufiger vor als man nach den relativ spärlichen Veröffentlichungen erwarten sollte. Laudenheimer hat mehr als 50 derartige Fälle aus dem Material der Leipziger psychiatrischen bezw. medicinischen Klinik, sowie aus der Privatpraxis zusammengestellt. Er betont die Schwierigkeiten der Diagnose, da die $\mathrm{CS}_{2}$-Intoxication keine typischen Bilder mache. Er unterscheidet

I. Allgemeine somatische Störungen.

II. Nervöse Störungen.

III. Psychische Störungen. 
Gemeinsam ist allen Formen eine Art Prodrome, bestehend in Schläfenweh, gastrischen Beschwerden, Schwindel und Müdiglkeit in den Beinen.

ad II sind peripherische (neuritische) Affectionen - hervorgerufen durch directen Contact der Hautoberfläche mit den flüssigen $\mathrm{CS}_{2}$ - abzutrennen von den eigentlichen, durch Einathmung des gasförmigen Giftes bedingter $\mathrm{CS}_{2}$ Neurosen. Letztere bilden sehr mannigfaltige Krankheitsbilder, nähern sich. namentlich oft dem Bild der Neurasthenie, von der sie sich jedoch durch die acute Entstehung und das vorwiegende Befallensein der unteren Extremitäten (Schwäche bis Parese der Beine, auch Pseudo-Tabes!) unterscheiden.

ad III berichtet Vortragender über 18 innerhalb 13 Jahren zur Beobachtung gekommene Fälle von Psychosen, in denen die toxische Aetiologie mit Sicherheit zu erweisen war. Oefters kamen gruppenweise Erkrankungen in dem Vulkanisirraume einer einzelnen Fabrik im Anschluss an local-temporäre Schädlichkeiten vor. Eine gewisse Disposition des Individuums scheint zum Züstandekommen einer geistigen Störung durch $\mathrm{CS}_{2}$ erforderlich, in schweren Fällen fand sich stets hereditäre Belastung. Die Psychose bricht durchschnittlich 3 Wochen nach Beginn der Vulkanisirarbeit aus. Arbeiter, die in den ersten 2 Monaten nicht afficirt werden, bleiben in der Regel dauernd psychisch gesund.

Laudenheimer unterscheidet folgende Frormen von Geistesstölung:

a) Maniakalische Formen, im Allgemeinen der reinen Manie (ohne Hallucinationen) entsprechend. Auffallend ist, dass sich intermittirend in dem manischen Verlaufe härfig kurze hypochondrische Episoden einschieben. Von somatischen Begleiterscheinungen kommt Tremor regelmässig, Pupillendiferenz bäufig vor. Ausgang nach durchschnitlich $2 \frac{1}{2}$ monatlicher Dauer stets in Heilung.

b) Depressive Formen. Hier kommt einfache melancholische Verstimmung nicht vor, sondern vorwiegend ein dem depressiven hallucinatorischen Wahnsinn entsprechendes Kranliheitsbild. Unter 10 Kranken dieser Gruppe wurden 4 unheilbar (Ausgang einmal in cbronisches hallucinatorisches Irresein, zweimal in chrouischen Wabnsinn mit Uebergang in Demenz, einmal in chronische Verrücktheit). Intercurrent auftretende Apathie und Benommenheit scheint von übler Vorbedeutung zu sein. Dauer der heilbaren Fälle ca. 3 Monate.

c) Stuporöse Formen. 1. Katatonisch-hebephrenische Krankheitsbilder, von schlechter Prognose. 2. Acuter heilbarer Stupor (= Kräpelin's Dementia acuta). Weite und Trägheit der Pupillen ist für die ganze Gruppe c charakteristisch.

d) Einfache Demenz lrommt namentlich nach lange andauemder, aber wenig intensiver Giftwirkung vor. Die Gedächtnissschwäche ist häufig irreparabel.

e) Charakterveränderung im Sinne eines moriatischen Wesens.

Die Therapie beschränkt sich auf allgemeine Indicationen. Viel wichtiger ist die Prophylaxe, bezüglich deren Laudenheimer eine Reihe gewerbehygienischer Maassnahmen vorschlägt. In Leipzig ist im Anschluss an 
hygienische Verbesserungen die psychische Morbidität der Gummiarbeiter bedeutend gesunken. Lamit jedoch nicht durch kostspielige Neueinrichtungen die Concurrenzfähigkeit einzelner Industriebezirke benachtheiligt werde, ist reichsgesetzliche Regelung der Angelegenheit erforderlich.

(Ausführliche Veröffentlichung erfolgt später.)

VIII. Herr G. Köster-Leipzig. Experimenteller und pathologisch-anatomischer Beitrag zur Lehre von der chronischen Schwefelkohlenstoffrergiftung.

Vortragender macht über die bis jetzt gewonnenen klinischen und anatomischen Resultate seiner an Kaninchen rorgenommenen chronischen Vergiftungen mit $\mathrm{CS}_{2}$ vorläufige Mittheilung. Das Gift wurde auf dem. Wege der Athmung der Blutbahn der Thiere einverleibt. Kilinisch konnte beobachtet wcrden: Dauernde Gewichtsabnahme, Erhöhung und von der 6. bis 7. Woche Herabsetzung der elektrischen Erregbarkeit unter Auftreten yon Ermüdungsreaction, Hyperästhesieen und bei 2 Thieren nicht auf bestimmte Nerren beschränkte Anästhesieen. Ferner wurden $4 \mathrm{mal}$ danernde Pupillenerweiterungen, 1 mal Pupillendifferenz, 2 mal dauernde Paresen der Extremitäten erzielt. Die Anfangs stark erregten Thiere wurden schliesslich stuporös. Somit wurden zuerst Reizerscheinungen, späterhin Ausfallserscheinungen beobachtet.

Die Untersuchung der sogleich post mortem geöffneten Thiere ergab makroskopisch nichts Besonderes, auch mikroskopisch keine Verfettung der inneren Organe. Auch Marischeidenzerfall wurde nirgends gefunden, wohI aber deutliche Verfettung vieler Zellen des gesammten Centralnerrensystems einschliesslich der Spinal- und Sympathicusganglienzellen.

Ferner wurde das gesammte Centralnervensystem mit der Nissl-Heldschen Methode zum Theil in Serienschnitten bei allen verstorbenen Thieren untersucht und die verschiedenartigsten Degenerationszustände an den Zellen festgestellt.

Vortragender entwirft sodann auf Grund der bei jedem Thier erhobenen Befunde das anatomische Bild der einzelnen Stadien der Zelldegeneration. Im Allgemeinen ronnten an den Zellen des gesammten Centralnervensystems, der Spinalganglienzellen und Sympathicusganglienzellen Veränderungen des Kerns (Schrumpfungen, Auflösungen, Verlust oder Quellung des Kernkörpers, Spaltbildungen, hyaline Entartung, Auflösung), Zerbröckeln und Abreissen der Dendriten, sowie Erweiterungen des pericellulären Raumes und Abreissen der den Zellleib umspinnenden Axencylinderfüsse beobachtet werden.

Die verschiedensien Degenerationscombinationen werden in ihren Haupttypen geschildert und an Zeichnungen erläutert. Besonderen Werth legt Vortragender auf die Thatsache, dass sich neben stark degenerirten Zellen annähernd oder ganz normale vorfinden. Vortragender vermuthet, dass auch dem Krankheitsbilde der chronischen $\mathrm{CS}_{2}$-Vergiftung des Menschen primäre Zelldegenerationen im Centralnervensystem zu Grunde liegen werden und weist darauf hin, dass auch beim Menschen vorwiegend Reiz- oder Ausfallserscheinungen beobachtet werden, dass das proteusartige Bild der $\mathrm{CS}_{2}-\mathrm{P}_{\mathrm{sy}}$ chosen sowie die schwere oder überhaupt unmögliche Heilung derselben ihren Grund 
in den verschiedenartigen Degenerationscombinationen der Zellen des Centralnervensystems haben könnte.

(Der Vortrag erscheint im Neurologischen Centralblatt.)

IX. Herr Stintzing-Jena. Zur Lehre des Tetanus.

Vortragender berichtet über einen schweren Fall von Tetanus traumaticus, der trotz Behandlung mit Behring'schem und danach mit Tizzonischem Antitoxin tödtlich verlief. Allerdings hatte die Antitoxinbehandlung erst 4 Tage nach Beginn der ersten Kranlheitserscheinungen eintreten können. Bei mehrfach vorgenommener Spinalpunction fand sich eine Steigerung des Druckes, die Spinalflüssigkeit war wasserklar. Mit Spinalflüssigkeit geimpfte Mäuse wurden tetanisch; doch zeigte sich innerhalb zweier Tage eine rasche Abnahme der Toxicität. Durch Verimpfung von Blut, welches dem TetanusJranken durch eine kleine Venaesection entnommen war, konnte kein Tetanus erzeugt werden. Demnach scheint das Tetanusgift nicht auf dem Wege der Blutbahn im Körper verbreitet zu werden. Vielmehr gewinnt die Hypothese von der Verbreitung des Giftes längs der Nerven an Wahrscheinlichkeit. Vortragender empfiehlt auf Grund seiner Versuche die Anwendung der Spinalpunction bei Tetanus zu diagnostischen Zwecken und zum Studium der Pathogenese der Krankheit. Schliesslich berichtet er noch über weitgehende anatomische Veränderungen im Rückenmark seiner Patienten: Erweiterungen der intramedullären Gefässe, Blutungen im Dorsalmark und hochgradige Degenerationen der Ganglienzellen in den Vorderloörnern.

$X$. Herr Ilberg-Sonnenstein. Die Bedeutung der Katatonie.

Vortragender legt grossen Werth auf die Anerkennung der von Kahlb a um entdeckten Katatonie als selbstständiger Krankheitsform, da die hierher gehörigen Fälle nach Symptomen, nach Art der Krankheitsentwickelung, nach Zeit des Beginns, Verlauf und Ausgang weitgehende Uebereinstimmung zeigen und durchans nicht selten sind.

Er skizzirt zunächst von den katatonischen Symptomen die Haltungs- und Bewegungsstereatypien, den Negativismus, Echopraxie, die auffallenden Gewohnheiten beim Essen, die Spraclanomalien (Mutacismus, Verbigeration, Ausstossen unarticulirter Töne, Echolalie, phrasenháfte Vielrederei und Sprachenverwirrtheit) and demonstrirt katatonische Schriftstücke, die die von $\mathbb{N}$ eisser geschilderten Eigenthümlichkeiten aufweisen. Katatonische Symptome sind für die Diagnose der Krankheit Katatonie conditio sine qua non, kommen aber ausserdem vor bei angeborenem oder secundärem Schwachsinn, bei periodischer Seelenstörung, Amentia, Hysterie, Dementia paranoides und progressiver Paralyse. Sehr wichtig ist die Art der Krankheitsentwickelnng in Form wechselnder Zustandsbilder, so zwar, dass entweder nach der Kahlbaum'schen Angabe dem Bild der Melancholie dasjenige der Manie, der Stupescenz, der Verwirrtheit und des terminalen Blödsinns folgen oder ein dagewesenes Stadium repetirt oder verschieden lange (event. Jalire bestehende) Remissionen zwischen den einzelnen Stadien liegen. Die Krankheit verläuft chronisch und geht oft rasch in geringeren oder höheren Schwachsinn über. Hallucinationen kommen nicht selten vor. In Sonnenstein starben 2 Katatoniker 18 bezw. 20 Jahre 
nach Beginn des Leidens; ein Kranker lebt noch dortselbst, der 40 Jahre Katatoniker ist. Im Gegensatz zu Kahlbaum und Schüle und in Uebereinstimmung mit Kräpelin hält Vortragender die Prognose der Kranliheit stets für schlecht. Der: körperlichen Begleiterscheinungen und der Krampfzustände wird gedacht.

20 vom Vortragenden beobachtete Fälle begamnen durchschnittlich im Alter von 24 Jahren, der früheste Fall entwickelte sich im 18., der späteste im 30. Lebensjahre. Die ursprüngliche Befähigung war bei der Hälfte der Fälle gut, bei der Hälfte mässig. Die Hälfte der Fälle waren Kopfarbeiter (Lehrer, Studenten, Kaufleute, Schreiber). 45 pCt. waren erblich belastet, davon nur 30 pCt. direct. Eine klare äussere Ursache der Krankheit ist nicht bekannt, 4. secirte männliche Gehirne hatten ein Durchschnittsgewicht von $1502 \mathrm{~g}$ (incl. weichen Hirnhäuten nnd $50 \mathrm{~g}$ Liquor cerebrospinalis), die Hemisphären waren gleich schwer. Das Gewicht des Hirnstammes verhielt sich zu dem des Kleinhirns, zu dem des Hirnmantels wie $10: 11: 79$. Die Hirnrinde zeigte mikroskopisch normale Gefässe und Nervenfasern; ein Theil der Ganglienzellen war atrophirt. Um viele Ganglienzellen lagen ganze Reihen von Neurogliakernen herum.

Obwohl die Hebephrenie und die Katatonie manche verwandtschaftliche Beziehungen haben, kann sich Vortragender für die Zusammenfassung beider differenter Irrankheitsformen, wie dies A schaffenburg vorschlägt, nicht erwärmen und bestreitet die Nützlichkeit der von Schüle vorgenommenen Unterordnung der thatsächlichen Katatoniefälle unter die primäre Demenz. Es giebt Cebergangsfälle zwischen Katatonie und Hebephrenie und zwischen Katatonie und der klinisch sehr wichtigen Dementia paranoides Kräpelin's.

Discussion: Herr Binswanger steht hinsichtlich der Katatoniefrage auf dem Boden der Schülo'schen Auffassung. Er rechnet die Katalonie zu den degenerativen Psychosen und findet die erbliche Degeneration überwiegend. Er ist deshalb überrascht über die relativ geringe Erblichkeitsziffer bei den Ilberg'schen Fällen.

XI. Herr Ziehen-Jena beschränkt sich in Anbetracht der vorgerückten Zeit auf eine kurze Mittheilung über eine neue Methode dex Entlarvung der Simulation einer halbseitigen hysterischen Taubheit bezw. Blindheit. Dieselbe beruht im Wesentlichen darauf, dass die Explorandin, welche beispielsweise linksseitige Taubheit vorgiebt, rorgesprochene Worte theils bei geschlossenem, theils bei offenem Ohr (in unregelmässigem Wechsel) nachzusprechen aufgefordert wird. Sie spricht dabei nur diejenigen nach, welche ihr bei offenem rechtem $\mathrm{Ohr}$ vorgesprochen werden. Nach etwa 10 bis 15 Worten fordert man die Explorandin auf, die porgesprochenen Worte zu wiederholen. Die Simulantin vermag nun entsprechend bekannten psychologischen Thatsachen sich nicht mehr zu erinnern, welche Worte sie nachgesprochen hat und welche nicht. Die Einzelheiten der practischen Durchführung der Methode sind in der ausführlichen Mittheilung (Monatsschrift fïr Psychiatrie und Neurologie) nachzolesen. 
XII. Herr IIösel-Zschadrass. Ueber einige seltene secundäre Veränderungen nach Herden in Insel und Thalamus opticus.

Vortragender demonstrirt Weigert-Präparate von einem Gehirn, welches einen Herd in der linkein Insel mit Uebergreifen desselben auf den Fuss des Stabkranzes der vorderen Centralwindung und einen zweiten Herd im 'Thalamus opticus aufwies, der dessen ganzes centrales Kernlager und dasjenige des lateralen Kerns in seiner hinteren Hälfte zerstört hatte. Von den secundären Veränderungen, die diese ein Jahr alten Herde hervorgerufen hatten, besprach Vortragender folgende, seines Wissens bisher noch nicht beobachteten Befunde.

1. Der vordere Thalamusliern war vollständig zum Schwund gebracht, der erste positive Fall von Schwrnd des vorderen Thalamuskerns. Mit Bezugnahme auf die Monakow'sohe Arbeit über die Rindenzone der Thalamuskerne trat der Vortragende in eine Kritik dieser Verhältnisse ein und sprach die Ueberzeugung aus, dass das Paracentralläppchen zwar nicht sicher als corticale Zone des vorderen Thalamuskerns anszuschliessen sei, dass aber wahrscheinlicherweise die hinteren Abschnitte der unteren Stirnwindung das corticale Rindencentrum seien. Die obere Stirnwindung glaubt Vortragender ausschliessen zu müssen.

2. Macht Vortragender auf die ganz auffallende Thatsache aufmerksam, dass trotz totalen Schwundes des Thalamuslierns und trotz des langen Bestehens des Leidens das Vicq-d'Azyr'sche Bündel erhalten war.

3. Zeigte er, dass das Forel'sche Feld $\mathrm{H}_{2}$ ebenfalls total secundär zum Schwund gebracht war. Am entwickelungsgeschichtlichen Präparat, das dieses Feld als isolirt markscheidenhaltig aufwies, erklärte er Verlauf, Ursprang und Ende des Bündels.

4. Demonstrirte er noch die Zerstörung des äusseren und centralen Kernlagers des Thalamus bei fast vollständigem, trotz einjährigem Bestehen des Leidens, Erhaltensein des Schleifenhaupttheils und gab der Versammlung anheim, zu entscheiden, ob nach diesem Befund die Lehre Monakow's von der Unterbrechung der ganzen Schleife im Thalamus opticus haltbar sei oder ob nicht vielmehr seine Auffassung ron dem directen Verlauf der Rindenschleife die richtigere sei.

(Der Vortrag wird in einer grösseren Arbeit des Autors veröffentlicht.)

XIII. Herr Hänel-Halle berichtet eingehend über einen Fall von Neuroganglioma myelinicum verum, das sich in Gemeinschaft mit anderen Geschwulstformen auf der Dura eines Mannes fand, welcher an Gehirntumor gestorben war. (Der Vortrag ist in dem Hitzig'sehen Jubelheft dieser Zeitschrift ausführlich veröffentlicht.)

XIV. Herr Buchbolz-Marburg demonstrirt eine Reihe von Photographieen und Röntgen-Aufnahmen:

1. Photographieen einer an Lues cerebri leidenden Frau mit einseitigem 
Graefe'schen Symptom. Die Krankengeschichte der Pat. ist von Dr. Wi ckel in dieser Zeitschrift Band 30, 2 veröffentlicht worden.

2. Photographieen der unteren Extremitäten einer an Tabes dorsalis leidenden Frau. Die ganze linke untere Extremität ist nach aussen rotirt, das linke Knie verdickt, der Unterschenkel verlängert, die Malleolen verdickt, die Knochen oberhalb der Malleolen dagegen stark verdünnt, so dass man hier ca. $10 \mathrm{Ctm}$. oberhalb der Fussgelenke den Unterschenkel mit den Fingern einer Hand umspannen kann. Der Fuss steht rechtwinklig gegen den Unterschenkel flectirt. Ausgesprochener Pes planus.

3. Röntgenaufnahmen dieselbe Kranke betreffend

a) beider Kniegelenke. An dem linken Knie macht sich die Aussenrotation dadurch auf das deutlichste bemerkbar, dass die Patella bei der Aufnahme von oben her ganz seitwärts sichtbar ist;

b) des Pes planus;

c) des verlängerten Unterschenkels; es tritt auf der Platte die Atrophie im unteren Ende der Tibia deutlich hervor gegenüber dem verdiokten oberen Ende der Tibia and dem verdickten Condylus des Oberschenkels.

4. Röntgenaufnahmen der beiden Füsse einer Frau, die in ihrer Kindheit an einer Poliomyelitis auterior gelitten hatte. Die ganze rechte untere Extremität ist im Wachstlsum zurückgeblieben, vor allem der Fuss, der in einen ausgeprägten Pes calcaneus verwandelt ist. Der Talus ist verkümmert, der Calcanens steht fast senkrecht, die kleinen Fusswurzelknochen sind dorsalwärts hervorgetrieben, Mittelfuss und Zehen sind erheblich an Grösse reducirt.

5. Röntgenaufnahme der beiden Hänđe eines jungen Mädchens, welche an cerebraler Kinderlähmung leidet. Die rechte Hand, sowohl Skelett als auch Weichtheile, ist erheblich kleiner als die linke. - Es war die ganze rechte Körperhälfte gegenüber der linken im Wachsthum zurückgeblieben.

Sodann berichtet Vortr. unter Demonstrationen von Präparaten und Zeichnungen über einen Fall von secundärem Gehirncarcinom, welches durch die ausserordentlich grosse Zahl der Carcinomknoten und durch den Sitz einzelner derselben ausgezeichnet ist.

(Der Vortrag ist in extenso in der Monatsschrift für Psychiatrie und Neurologie veröffentlicht.)

XV. Herr Matthes-Jena. Befund des Rückenmarks bei Poliomyelitis acuta.

Vortr. demonstrirt Rückenmarkspräparate eines 8 Tage nach Einsetzen der Poliomyelitis an Pneumonie verstorbenen Kindes.

Es fand sich eine erhebliche kleinzellige Infiltration im Vorderhorn und ausgedehnte Gefässveränderungen, das Bild einer diffusen alle Gewebselemente gleichmässig betreffenden Myelitis.

Die Ganglienzellen im Herd und in der Nähe des Herdes zeigten sich in der Form vielfach auffallend gut erhalten, gegenüber der Zertrümmerung der 
514 III. Versamml. mitteldeutscher Psychiater und Neurologen.

Nervenfasern. Nisslpräparte lassen aber erkennen, dass dieselben stark verändert sind.

Herr Binswanger verzichtet mit Rücksicht auf die vorgeschrittene Zeit anf die beabsichtigte Demonstration seiner pathologisch-histologischen Präparate (über Lympheirculation in der Grosshirnrinde und arteriosclerotische Hirndegeneration). Er beschränkt sich auf die Demonstration einiger makroskopischer Präparate und von Zeichnungen und hofft seine Mittheilungen in der nächsten Versammlung machen zu können.

Der Yorsitzende Herr Mayser schliesst die Versammlung um $4^{1} / 2$ Uhr.

Wie üblich, fand nachher ein Festmahl unter zahlreicher Betheiligung statt, an welches sich ein gemüthliches Zusammensein anschloss.

Krause (Jena). 